\title{
A Model for Human Skin Impedance During Surface Functional Neuromuscular Stimulation
}

\author{
Stephen J. Dorgan, Member, IEEE, and Richard B. Reilly, Member, IEEE
}

\begin{abstract}
A new mathematical model for the bulk electrical impedance of human skin is presented. In particular this model describes the impedance of skin during surface functional neuromuscular stimulation (FNS) with square stimulation pulses. Experimental data are presented that illustrate the nonlinear dynamic properties of human skin during current and voltage controlled stimulation. Model predictions are compared to experimental data, measured under both constant voltage and constant current transcutaneous stimulation. It is found that this model captures a variety of nonlinear time-varying effects observed in the skin impedance when stimulating with either protocol. This model may be used as part of large neuromusculoskeletal models or in the more accurate modeling of transcutaneous FNS, which is currently the most common clinical implementation of FNS.
\end{abstract}

Index Terms - Impedance, modeling, neuromuscular stimulation, nonlinear systems, skin.

\section{INTRODUCTION}

$\mathbf{F}$ UNCTIONAL neuromuscular stimulation (FNS) has been employed widely to artificially activate skeletal muscles and so partially return motor function to the spinal cord injured (SCI) [1], [11], [28]. Prosthetic and orthotic devices using FNS have been developed to restore a SCI individual's ability to stand, walk and grasp [1], [11]-[13], [28]. A variety of activation methodologies may be applied in situ to initiate neural action potentials and so provide the required force generation capability for these devices [14]. In a rehabilitation setting, rectangular constant current or voltage electrical pulses are typically applied transcutaneously to activate motor neurones and so initiate muscular contractions [1]. It has recently been shown that square subthreshold depolarising prepulses may be useful in closing the slow $\mathrm{h}$ gates in nerves to elevate the activation threshold of nerve fibers [7]. This may provide an effective strategy within FNS to disable superficial pain fibers and enable a larger range of motor neurones to be activated in incomplete SCI subjects [7], [25]. These applications involve overcoming the electrical impedance of the skin to activate the motor neurones underlying the surface. If an accurate model for the impedance of skin was known it may be possible

Manuscript received January 2, 1998; revised December 21, 1998 and June 3,1999 . This work was supported in part by the European Commission under the TMR programme.

S. J. Dorgan is with Lehrstuhl für Steuerungs-und Regelungstechnik, Technical University of Munich, Munich 80333 Germany (e-mail: s.j.dorgan@ieee.org). He is also with the Department of Electronic and Electrical Engineering, University College Dublin, National University of Ireland, Dublin 4 Ireland.

R. B. Reilly is with the Department of Electronic and Electrical Engineering, University College Dublin, National University of Ireland, Dublin 4 Ireland. Publisher Item Identifier S 1063-6528(99)07096-2. to design optimal stimulation waveforms to achieve more efficient nerve and so muscle activation, possibly with less pain induced.

While the characteristics of the skin's electrical impedance have been the subject of study under a variety of conditions [2], [15], [22], [29], the phenomena exhibited are still far from being well understood. Unlike muscular tissue resistivity, which has a linear characteristic for sinusoidal current densities below $2 \mathrm{mAcm}^{-2}$ in the frequency range $10 \mathrm{~Hz}$ to $10 \mathrm{kHz}$ [24], [27], [31], skin exhibits a number of amplitude and frequency dependent nonlinear effects. Many investigations have been performed to date to characterize the nonlinear dependence of skin's impedance during sinusoidal AC stimulation on both the frequency and amplitude of activation [6], [31], [32]. As a result, models for the skin's AC impedance have been developed [15], [19], [23]. The properties of individual model components and their relation to the physical structure of the system have also been identified [15], [18], [19]. Unfortunately, while both rigorous and comprehensive, the results of these $\mathrm{AC}$ investigations are of limited relevance to FNS applications, where the stimulation pulses applied transcutaneously are typically of lower frequencies, higher amplitudes and rectangular in shape. This is particularly significant as it has been shown that the skin's nonlinearities are most pronounced when using high currents at low frequencies, just as is the case in transcutaneous FNS [18], [19], [31], [32].

The qualitative nature of the skin impedance in response to rectangular stimulation pulses has previously been studied [2] and a linear equivalent electrical model has been developed [2], [16]. This model is shown in Fig. 1. The resistor $R_{s}$ primarily attempts to model the resistance of deep tissues, while the parallel combination of capacitor $C_{p}$ and linear resistor $R_{p}$, is intended to model the skin impedance [2], [16], [31]. A series capacitance $C_{\text {pol }}$ and resistance $R_{\text {pol }}$ modeling the electrode-skin interface may also be included. However, for square dc stimulation pulses the capacitance $C_{\text {pol }}$ is found to be negligible and the resistance $R_{\text {pol }}$ is included in $R_{s}$ [2]. The structure of Fig. 1(a) therefore reduces to the electrical equivalent circuit of Fig. 1(b). A number of simple methodologies for identifying the values of the elements in Fig. 1(b) have been proposed and validated [16]. As a result the qualitative properties of each component in this linear system model have previously been identified [2], [16], [31]. However, it has long been known that the impedance of skin in response to dc current pulses displays a variety of complex nonlinear behaviors which this linear model is unable to replicate [2], [16]. 


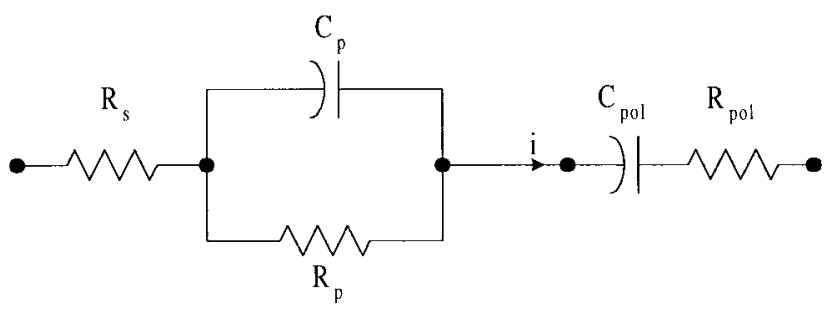

(a)

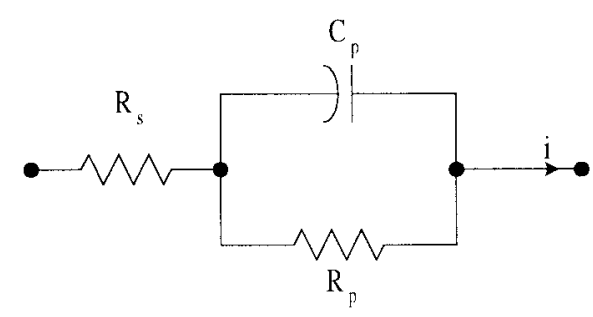

(b)

Fig. 1. Previous equivalent circuits to model skin and deep tissue [2], [16]. (a) General circuit, with $R_{s}$ primarily modeling the resistance of deep tissues, while capacitor $C_{p}$ and linear resistor $R_{p}$, model the skin impedance. The capacitance $C_{\mathrm{pol}}$ and resistance $R_{\mathrm{pol}}$ due to the electrode-skin interface are also shown. (b) Reduced circuit found sufficient to model large dc pulses.

This paper aims to present a new model for human skin that captures the system dynamics observed experimentally under both constant current and constant voltage stimulation with typical FNS pulses.

\section{MethodS}

All experiments were performed using a custom designed computer controlled electrical stimulation unit and a high speed PC based data acquisition system [20]. The stimulator used was capable of providing voltage controlled pulses in the range from 0 to $170 \mathrm{~V}$ and current controlled pulses in the range from 0 to $70 \mathrm{~mA}$, with the ability to vary the amplitude, pulse width (PW) and interpulse interval (IPI) of each pulse as desired. This stimulator was controlled by custom software via a high speed real-time interface card. For the experiments presented here the stimulator was made monophasic by removing the charge balancing network. The voltage applied across the stimulation electrodes was captured using a high speed digital storage oscilloscope connected to the PC, while the current flowing was similarly measured using a $1-\Omega$ precision resistor in series with the stimulator leads. These signals were periodically downloaded to the computer for offline analysis. All results presented here were obtained with self adhesive surface gel electrodes (Stimtrode Model ST75D) of dimensions $4.5 \mathrm{~cm} \times 2 \mathrm{~cm}$ as it's a typical electrode size for transcutaneous FNS.

The experiments presented for intact human skin were performed over the quadriceps muscle group of four ablebodied volunteers over the course of 30-40 experimental trials. In order to guarantee uniform electrode-skin contact the site under the electrode was shaved. No other chemical or cosmetic preparation of the electrode site was performed. To ensure that the nonlinear behaviors observed were truly independent of the current direction experiments were repeated

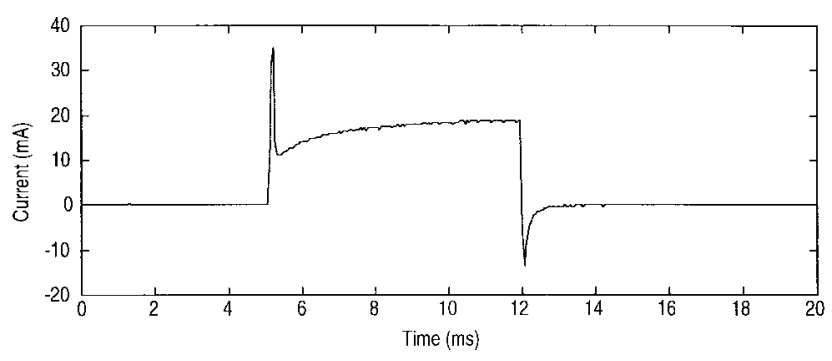

(a)

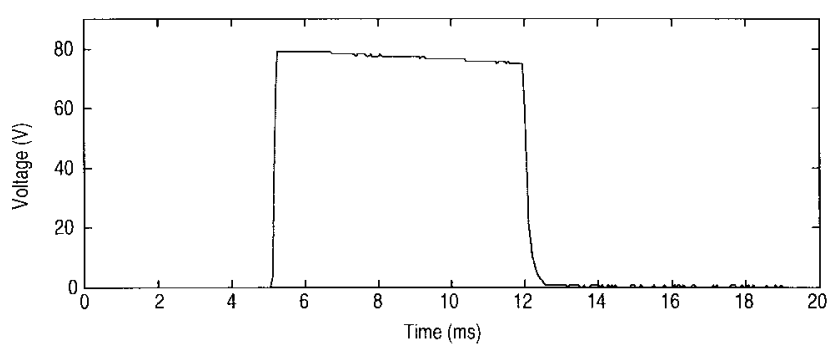

(b)

Fig. 2. (a) Current passing through the skin in response to (b) the constant voltage stimulation pulse.

with current flowing in the opposite direction. Experiments were also performed using two other types of electrodes from other manufacturers. In both cases no significant differences were found in the dynamics observed. In the experiments performed to investigate the effect of removing the upper layer of dead skin, known as the stratum corneum, the sites under the electrodes were lightly abraded with emery paper.

\section{MODEL DEVELOPMENT}

\section{A. Properties of Skin}

While inadequate, the simple electrical circuit in Fig. 1(b) proves a useful starting point for the analysis of the skin's response to an electrical stimulus. Fig. 2 shows the current flowing through the complete system when an $80-\mathrm{V}$ voltage controlled stimulation pulse of PW $6.8 \mathrm{~ms}$ is applied. The resistance $R_{s}$ in Fig. 1(b) can be identified during constant voltage stimulation by dividing the applied voltage by the peak current flowing, as the capacitor is momentarily shorting the resistor $R_{p}$. Similarly, at the end of a stimulation pulse of sufficient width the same calculation yields a value for $R_{s}+R_{p}$, when the capacitor is fully charged. This in turn permits the estimation of $R_{p}$. Previous experimental evidence has shown that for untreated skin the value of $R_{s}$, which models resistance of the body core (and to a lesser extent elements of the stratum corneum and lower epidermis) is independent of the stimulation voltage, while there is an inverse relation between $R_{p}$ and the stimulation voltage [2], [21], [22]. As may be seen in Fig. 2 there is a renewed increase in the current flowing after the initial drop. Such a late increase is in agreement with the phenomena observed in previous qualitative studies [10], [22]. Van Boxtel [2] also found a similar depression in the current through the skin after the initial capacitive spike before it returned to the steady 


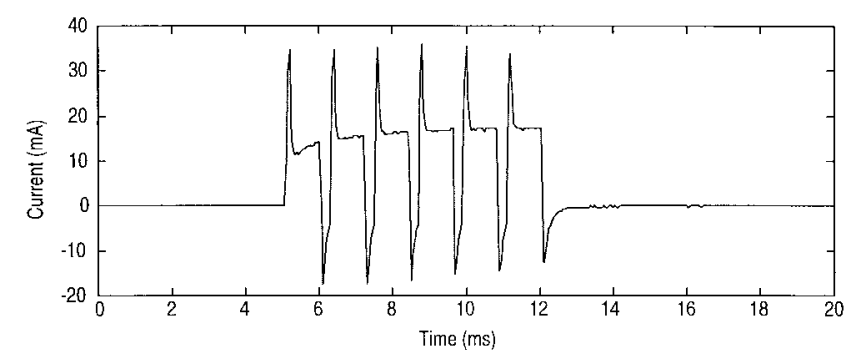

(a)

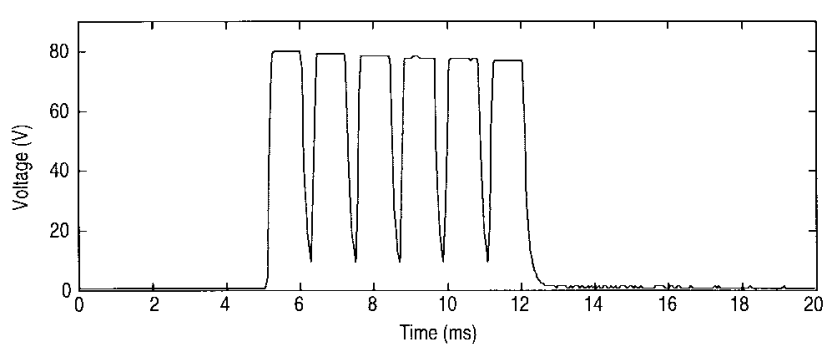

(b)

Fig. 3. (a) Current passing through the skin in response to (b) the train of constant voltage stimulation pulses.

state value. At the end of the voltage pulse there is a quasisteady-state relationship between the voltage applied and the current flowing as shown in Fig. 2. The term "quasi" is used as the current is still increasing at a very low rate at the end of the pulse. It is also worth noting that the pulse widths considered here are considerably larger than those typically employed during FNS. Therefore a model capable of capturing the dynamics over this longer time scale will certainly capture the dynamics during the shorter more typical FNS stimulation pulses.

Removal of the stratum corneum is shown to reduce the value of $R_{s}$, while almost eliminating $R_{p}$ and the system's nonlinearities completely [2]. This demonstrates that in intact skin the voltage or current dependent impedance of intact skin modeled by $R_{p}$ resides almost exclusively in the stratum corneum. In particular it has been shown that ionic conduction within the stratum corneum is the main cause of the skin's nonlinear impedance properties [31], [32], and this premise forms the basis for the model proposed here.

\section{B. Model Proposed}

Fig. 3 shows the skin's response to a burst of six shorter pulses, each of PW 1ms. These experimental results would appear to indicate the presence of some sort of memory element within the impedance considered. While the response to a single voltage controlled pulse, as in Fig. 2, may be modeled by a simple resistor-capacitor network, such as that in Fig. 1(b), the response presented here to a train of six pulses cannot. Such a memory effect could be incorporated, in part, into the model in Fig. 1(b) by introducing an inductive element. Yet, while resistive and capacitive sources abound in the system under consideration there is no obvious physiological source for an inductive component in the body. This is an important consideration if the model is to reflect the physio-

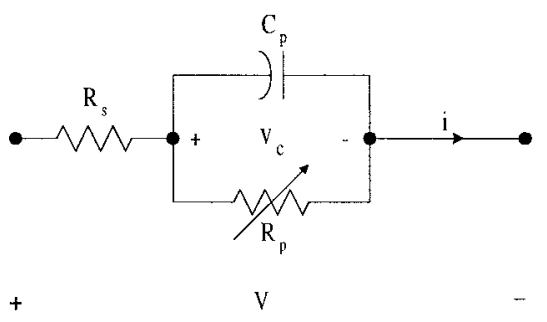

Fig. 4. Skin impedance model proposed here. Resistance $R_{s}$ models the constant resistance components of the skin and deep tissue resistance. Variable resistor $R_{p}$, with capacitor $C_{p}$, serves to model all the nonlinear history dependent dynamics of the skin impedance.

logical reality and be of use (with larger neuromusculoskeletal models) in the design of optimal FNS stimulation strategies or pulses for disabling superficial pain fibers. However, models of ionic conduction through biological membranes, such as the well-known Hodgkin-Huxley $(\mathrm{H}-\mathrm{H})$ nerve model [8], [9], [30], which contain only resistive and capacitive elements do exhibit history dependent (i.e., memory) effects.

The system proposed here to model the skin bulk dc impedance is shown in Fig. 4. It consists of the parallel arrangement of a constant linear capacitor, $C_{p}$, with a nonlinear time-dependent resistor $R_{p}$, which is in series with a constant linear resistor $R_{s}$. Modeling the memory component of the impedance, demonstrated in Fig. 3, is achieved by employing a modified form of the H-H equations [9], [30] to describe $R_{p}$.

While such a model is similar to that used to capture the behavior of a single ionic conduction channel [8] its basic structure bears a similarity to the model proposed previously (Fig. 1) [2], [16]. However, unlike the previous models developed, all the nonlinear characteristics of the impedance within this model lie in $R_{p}$. In this way experimental findings that the system nonlinearities cannot be attributed to $C_{p}$ or $R_{s}$ [31] are incorporated into the proposed model for $R_{p}$. The precise equations used to describe $R_{p}$ are

$$
\begin{aligned}
R_{p} & =1 / g_{p} \\
g_{p} & =\frac{1}{\tau_{g}}\left[g_{p \infty}-g_{p}\right]+g_{x} \\
\frac{1}{\tau_{g}} & =\beta_{g}+\alpha_{g}\left(c_{1}-\beta_{g}\right) \\
g_{p \infty} & =\frac{g_{q p}(i) \cdot g_{p k}}{g_{p k}-g_{q p}(i)} \\
g_{q p}(i) & =\lambda_{1}+\lambda_{2} \cdot\left(1-e^{\lambda_{3} i}\right) \\
g_{x} & =\frac{1}{\tau_{x}}\left[g_{\text {ch }}+g_{i} i-q\right]
\end{aligned}
$$

where $g_{p}$ and $g_{s}$ are the conductances of $R_{p}$ and $R_{s}$, respectively. $g_{p \infty}$ is the quasi-steady-state value of $g_{p}$ at the end of the stimulation pulse and $g_{q p}(i)$ is the function modeling the variation of the quasi-steady-state conductance as a function of the injected current, $i$. The injected charge is $q$, while $g_{x}$ models the time and charge dependent variation of the conductance $g_{p}$ from the constant value of $g_{\mathbf{c h}}$. The variation of $g_{q p}$ with the current injected found in experiment is shown in Fig. 5, as is the function $g_{q p}(i)$ identified using the Levenberg-Marquardt nonlinear least squares method [17]. The quantities $\alpha_{g}$ and $\beta_{g}$ 


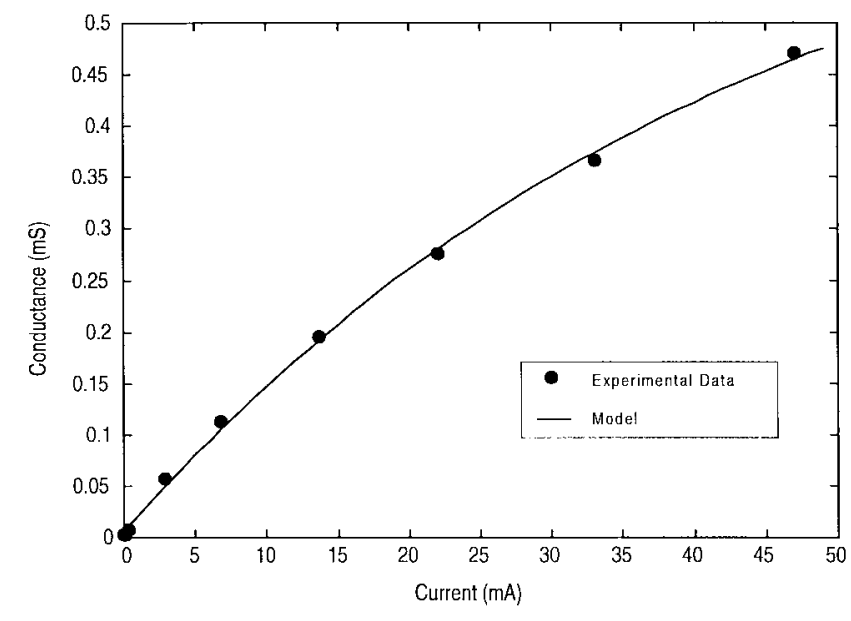

Fig. 5. Variation in the conductance $g_{q p}$ identified in experiment (filled circles) and the function $g_{q p}(i)$ identified to fit the data according to a least squares criterion (solid line).

in (3) determine the nonlinear current dependent dynamics of the conductance $g_{p}$ and are

$$
\begin{aligned}
\alpha_{g} & =c_{2} \cdot\left(1-e^{c_{3}\left(v+v_{r}\right)}\right) \\
\beta_{g} & =\frac{c_{4}}{e^{c_{5}\left(v+v_{r}+c_{6}\right)}+1}
\end{aligned}
$$

while $v$ is the voltage across the electrodes and $v_{r}$ models the half cell (or contact) potentials that occur at surface interfaces, $i_{0} \in \Re$ and $c_{i} \in \Re \forall i$. For constant current stimulation the system model is precisely the same, the only difference being that the current $i$ forms the model input and the voltage $v$ then becomes the quantity calculated.

A mathematical model for the skin impedance during FNS has been presented in (1)-(8). The model structure is a significantly reduced form of the $\mathrm{H}-\mathrm{H}$ equations, that possesses the desired property of history dependence, which is maintained by the presence of the first-order lag, without the complex structure.

\section{RESULTS}

The model equations described in Section III have been implemented using a commercial simulation package and are readily simulated using a third-order Runge-Kutta integration method, which utilizes a second-order method for step size control. The sole inputs to the model system are perfectly rectangular voltage or current controlled stimulation pulses of the same pulse widths and amplitudes used in experiment. The parameters presented in the Appendix were used for all the simulations shown. These intact skin parameters found in Table I were identified using data other than that presented here (but which was recorded during the same experiment) in order that the system be validated against an 'unseen' data set. The determination of parameters to describe abraded skin is discussed later.

\section{A. Response to a Constant Voltage Sequence}

Fig. 6 illustrates a simulation of the model system carried out to ascertain whether the impedance structure proposed

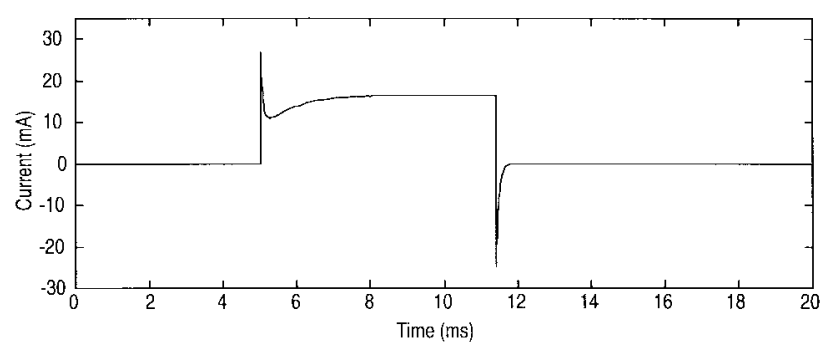

(a)

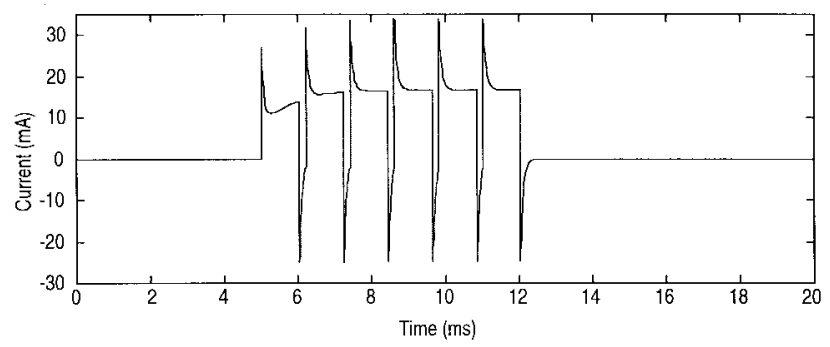

(b)

Fig. 6. Current passing through the skin as predicted by the model for (a) a single long pulse and (b) a burst of six shorter ones when using a $\mathrm{H}-\mathrm{H}$ like impedance to model the skin.

in Section III and illustrated in Fig. 4 is a viable model. the experiments presented in Figs. 2 and 3 were repeated in simulation. Such a test is significant for the model as its primary feature: the time dependent nature of the $R_{p}$ conductance, is being presented as an alternative approach to describing the skin impedance presented to the stimulator. In comparing the simulation results in Fig. 6 with the experimental results in Figs. 2 and 3 it may be seen that the model appears to capture the dynamics observed experimentally, both qualitatively and quantitatively. These results indicate that the model realizes successfully the 'memory' effect evident in the experimental data shown in Fig. 3.

As suggested by previous experimental evidence [2], [16], [31], the initial current spike in the model is a capacitive current as a result of the fast rising edge of the voltage pulse. The subsequent increase in the current after this capacitive spike occurs due to a reduction over time in the value of the parallel resistor $R_{p}$.

\section{B. Intact Skin}

Fig. 7 shows the measured current flowing through the system in response to voltage controlled stimulation pulses applied in $10 \mathrm{~V}$ intervals in the range from 10 to $100 \mathrm{~V}$. As can be seen there is a nonlinear increase in both the maximum current amplitude and the dynamics of the system. Such a response is similar to those observed by others experimentally [2], [22]. Shown in Fig. 8 are the responses predicted by the model when using the parameters presented in Table I and voltage stimulation pulses of the same amplitudes and PW as in experiment. In comparing the simulation results of Fig. 8 with the experimental results in Fig. 7 it may be seen that the model successfully predicts both the quantitative and qualitative behavior of the system at different amplitude levels under constant voltage stimulation. 


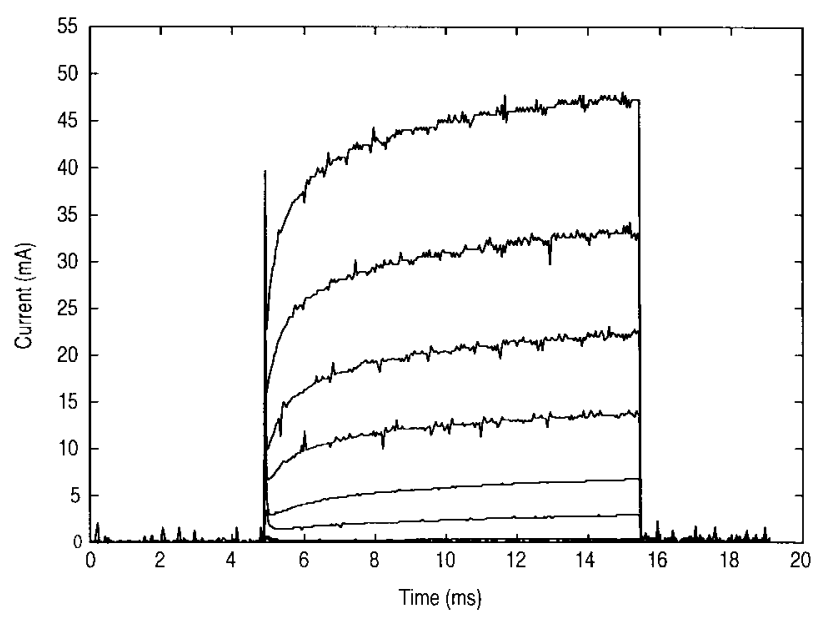

Fig. 7. Experimentally measured current waveforms in response to constant voltage stimulation. The stimulation voltage pulses ranged from 10 to $100 \mathrm{~V}$ in $10-\mathrm{V}$ steps. The responses under $50 \mathrm{~V}$ may just be seen above the $x$-axis.

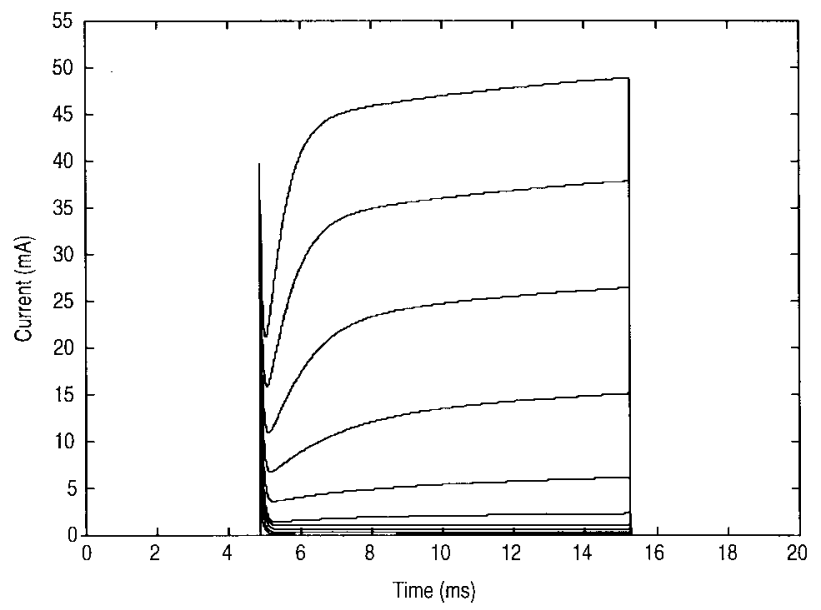

Fig. 8. Simulated current waveforms in response to constant voltage stimulation pulses ranging in amplitude from 10 to $100 \mathrm{~V}$ in $10-\mathrm{V}$ steps when using the model proposed with the parameters in Table I.

An important test for this model, which was developed and identified for constant voltage stimulation, is how it performs when its predictions are compared to the system's responses under constant current stimulation. Fig. 9 shows the system's voltage responses measured in experiment to current controlled stimulation inputs of $0.14,0.818,2.3,8.09,14.36$, and $18.54 \mathrm{~mA}$. These stimulation current values are shown to illustrate the diverse range of responses observed with constant current stimulation at various current levels. They also serve to illustrate the difference between the system dynamics observed under constant current stimulation and those observed under constant voltage stimulation. Notice in particular the apparent decay in the system voltage at higher currents. This decay has been observed by others [25] and has been identified as a dynamic property of the system. Constant current stimulation pulses were used as inputs to the model, again using the parameters presented in Table I of the Appendix. Fig. 10 shows the responses predicted by the model for this activation.

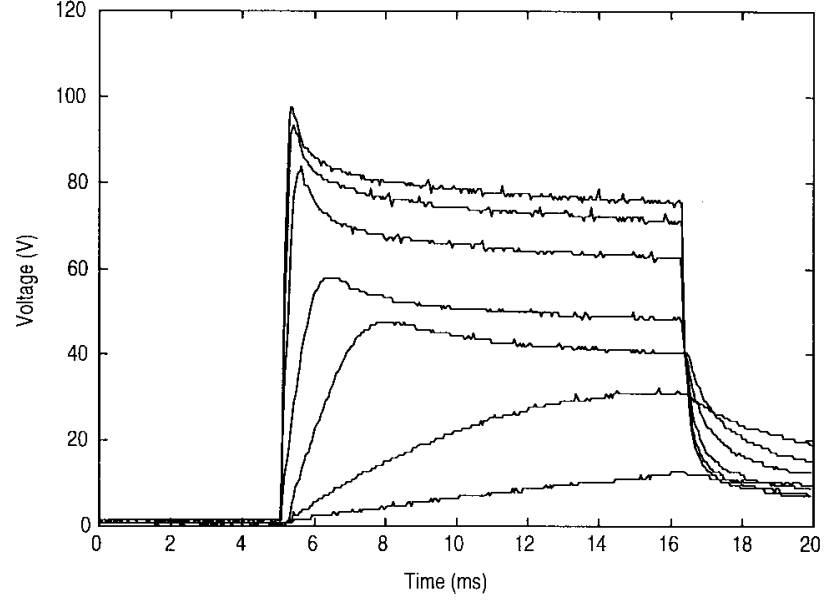

Fig. 9. Experimentally measured voltage waveforms in response to constant current stimulation pulses of magnitude $0.14,0.818,2.3,8.09,14.36$, and $18.54 \mathrm{~mA}$.



Fig. 10. Simulation voltage waveforms predicted by the model, when using the parameters in Table I, for perfectly square constant current stimulation of magnitude $0.14,0.818,2.3,8.09,14.36$, and $18.54 \mathrm{~mA}$.

From Fig. 10, it may be seen that the model appears to capture both the variation of the voltage amplitude with stimulation level and the decay in voltage with time. However, the initial voltage "spike" appears to be larger in simulation than it is in experiment. This may be explained by the fact that perfectly square stimulation pulses, which are not achievable in reality, were used in simulation. The constant current stimulation pulses applied in experiment were slightly rounded, similar to the voltage pulse illustrated in Fig. 2. In an effort to confirm that this is actually the case, similarly slightly rounded current pulses were used as inputs to the model in simulation and the voltage evoked was calculated. The results of these simulations are shown in Fig. 11, from which it may be seen that the model does indeed also capture the variety of effects observed during constant current activation of the system.

\section{Abraded Skin}

In order to test the physiological predictive nature of the model proposed, the system being considered was physically 


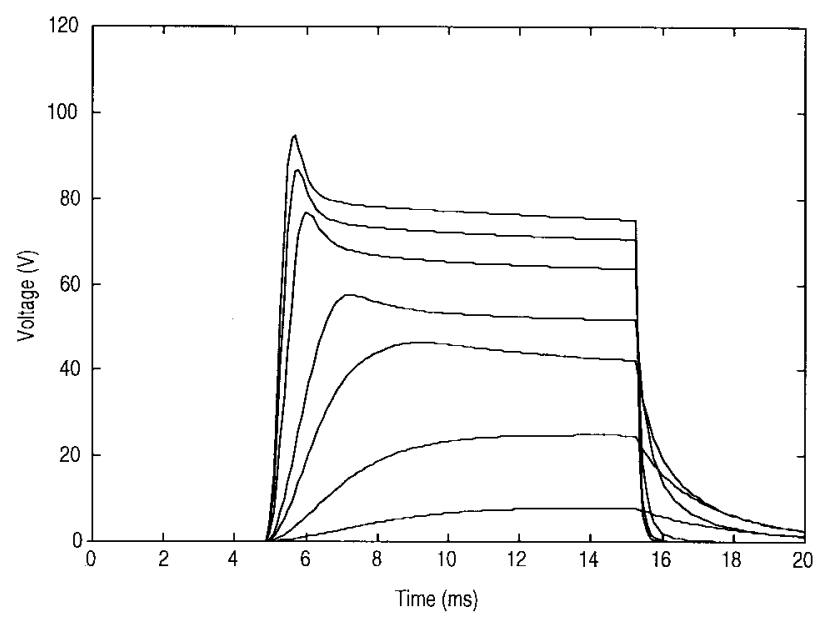

Fig. 11. Simulation voltage waveforms predicted by the model for constant current stimulation of magnitude $0.14,0.818,2.3,8.09,14.36$, and $18.54 \mathrm{~mA}$ where the input current pulses have been rounded to reflect those identified in experiment. Parameters again as in Table I.

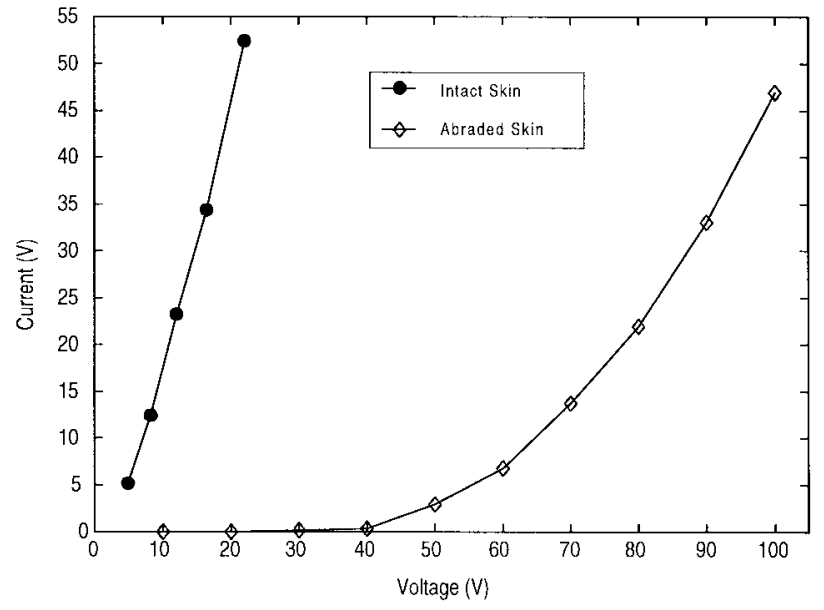

Fig. 12. Stimulation voltage magnitudes required to achieve the same end of pulse current magnitudes for intact skin (closed circles) and abraded skin (open diamonds).

altered. The parameters in the model changed to capture these new system dynamics were then investigated. If the model is a reasonable representation of the physiological system one would expect the resulting parameter changes to illustrate in some way the physical changes that occurred in the system.

Similar sites with identical electrode spacing, type, and size as in Section IV-A and IV-B were used. However, in this instance, the skin was also abraded with fine grade emery paper. This was performed to remove the upper layer of skin, the stratum corneum. The stimulation voltage levels required to obtain approximately the same current flow at the end of a stimulation pulse through the system as in Fig. 7 were identified and are shown in Fig. 12. Also shown is the currentvoltage relationship for intact skin identified previously in the experiment. As can be seen both the impedance and the degree of nonlinearity in the system has been significantly reduced by removing the stratum corneum, as was expected from previous studies [2], [16], [22]. These voltage levels were

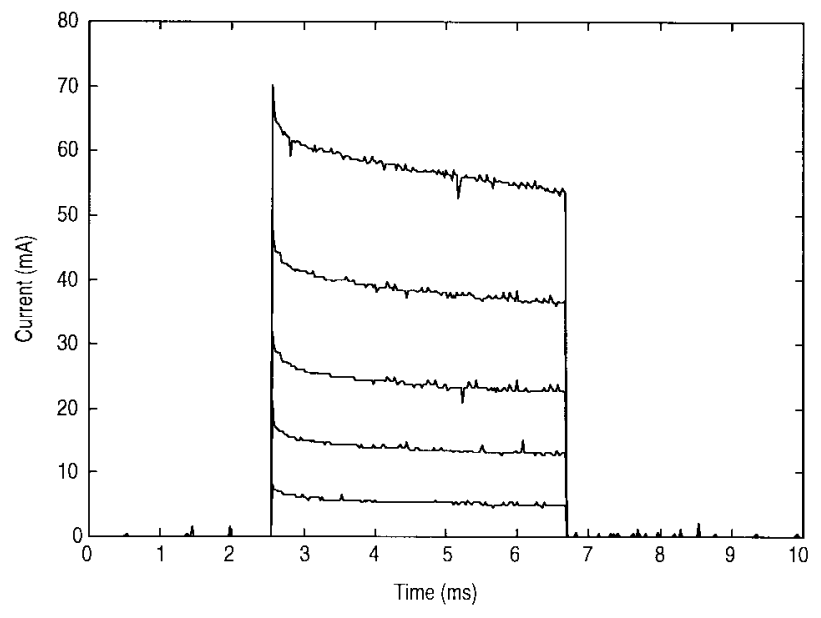

Fig. 13. Experimentally measured current flowing through abraded skin in response to constant voltage stimulation. Stimulation voltage magnitudes of $5,8.2,12,16.5$, and $25.8 \mathrm{~V}$ were used so that the current magnitudes at end of each pulse is similar to that shown in Fig. 7.

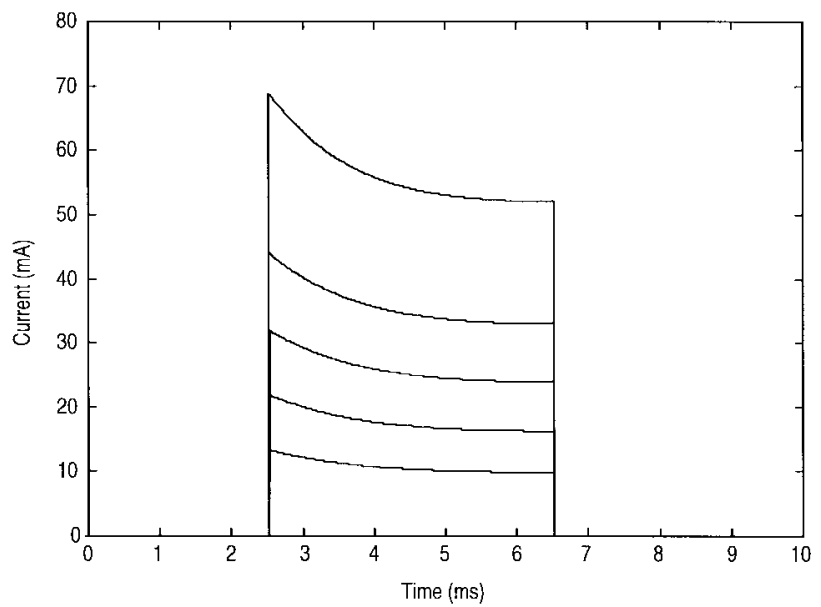

Fig. 14. Simulated current flowing through the model of abraded skin, with the parameters in Table II, in response to constant voltage stimulation at 5 , $8.2,12,16.5$, and $25.8 \mathrm{~V}$

then used as inputs to the abraded system. The set of current waveforms through the abraded skin in response to these lower stimulation voltages is presented in Fig. 13. From these results it may be seen that by removing the stratum corneum many of the nonlinear dynamics observed in nonabraded skin and shown in Fig. 7 are significantly reduced. In Fig. 14 the results of simulations carried out with the same model structure as previously are presented. The parameter values required to model the system with now abraded skin are presented in Table II of the Appendix. From these parameters it may be seen that reductions in the values of parameters $R_{p}, R_{s}$, and $C_{p}$ are required to model the now altered system. In particular, it is noticed that the values of $C_{p}$ and $R_{s}$ are reduced to 50 and $25 \%$, respectively, of the values for modeling intact skin. Similarly, $v_{r}$ is reduced from 42 to $10 \mathrm{~V}$ and $R_{p}$ is effectively reduced to zero. These changed parameters reflect accurately both the changes the system has undergone and are of the same order reported in previous qualitative studies [2], [16], [32]. 
TABLE I

Simulation Parameters for Intact Human Skin

\begin{tabular}{c|c|c|c}
\hline VARIABLE & VALUE & VARIABLE & VALUE \\
\hline$\lambda_{I}$ & $5.3 \mu \mathrm{S}$ & $c_{5}$ & 10 \\
\hline$\lambda_{2}$ & $0.7028 \mathrm{mS}$ & $c_{6}$ & 0.1 \\
\hline$\lambda_{3}$ & -0.02256 & $\tau_{x}$ & $l$ \\
\hline$c_{l}$ & 1.35 & $g_{c h}$ & $0.05 \mathrm{mS}$ \\
\hline$c_{2}$ & 4.64 & $g_{p k}$ & $3.065 \mathrm{mS}$ \\
\hline$c_{3}$ & $0.03 / 25$ & $v_{r}$ & $-42 \mathrm{~V}$ \\
\hline$c_{4}$ & 1.35 & $g_{s}$ & $3.065 \mathrm{mS}$ \\
\hline- & - & $C_{p}$ & $0.02358 \mu \mathrm{F}$ \\
\hline
\end{tabular}

\section{DISCUSSION}

The control of FNS musculoskeletal systems is of particular interest to those using FNS in a rehabilitation setting. A central element in the design of control strategies is the possession of a good mathematical model for the system to be controlled. A mathematical model for human skin impedance has been developed and presented. This model while conceptually simple is nonlinear and time-varying, as is the real physiological system. The nonlinearities within this system are modeled as primarily occurring due to ionic conduction within the stratum corneum, as has been shown to be the case in experiment [31]. The system equations are compact, robust, numerically stable and easily implemented within most simulation packages. This model may be combined with others [3], [4], [5], [26] to form a more complete model of the neuromusculoskeletal system when activated under transcutaneous FNS. It may also be of use in the design of optimal FNS stimulation strategies for the reduction of pain [7], [25], or "intelligent" controllers.

The results presented in Figs. 8-14 demonstrate the ability of this model to capture both qualitatively and quantitatively the variety of nonlinear time-varying effects characteristic of human skin's electrical impedance. This has been demonstrated under both constant voltage and constant current stimulation conditions so illustrating the ability of this model developed for constant voltage stimulation to also "predict" the effects observed during constant current stimulation.

Both the experimental and simulation results presented also suggest that removal of the stratum corneum greatly reduces the influence of the parallel $C_{p}, R_{p}$ network shown in Fig. 4 on the injected current during voltage stimulation. This is in agreement with the experimental phenomena previously documented by others [2], [16]. It would appear, from the changes in the model parameters required to capture the observed effects, that this is due to a reduction in the value of $R_{p}$ which effectively shorts the smaller skin capacitance $C_{p}$. Nevertheless, this model, as with any other, is limited by the mechanisms built into it. While this model captures a number of behaviors it may be expanded or extended further. This may be achieved, at the expense of increased complexity, by adding a clearly identifiable electrode model, or by making this essentially unidirectional model bidirectional.
TABLE II

Simulation Parameters For Abraded Human SKin

\begin{tabular}{c|c}
\hline VARIABLE & VALUE \\
\hline$g_{c h}$ & $0.03 \mathrm{mS}$ \\
\hline$g_{p k}$ & $0.766 \mathrm{mS}$ \\
\hline$v_{r}$ & $-10 \mathrm{~V}$ \\
\hline$C_{p}$ & $0.0125 \mu \mathrm{F}$ \\
\hline
\end{tabular}

It is also worth noting that from the experimental results presented that it appears considerable savings could be made both on the technology used to achieve the reduced voltages required and on the energy consumption of a stimulator if the system properties could be changed by a less aggressive and less irritating method other than removal of the stratum corneum. Perhaps this will become possible with a new generation of conductive gels.

\section{APPENDIX I}

Presented in Table I are all the parameter values necessary for the simulation of the skin impedance model for intact human skin presented in the text. These parameter values have been determined from experiments over the quadriceps muscle group of able bodied volunteers.

Presented in Table II are the parameter changes necessary for simulation of the skin impedance model for abraded human skin. All other parameters are as in Table I.

\section{REFERENCES}

[1] C. Billian and P. H. Gorman, "Upper extremity applications of functional neuromuscular stimulation," Assist. Technol., vol. 4, pp. 31-39, 1992.

[2] A. van Boxtel, "Skin resistance during square-wave electrical pulses of 1 to $10 \mathrm{~mA}$," Med. Biol. Eng. Comput., vol. 15, pp. 679-687, 1977.

[3] S. J. Dorgan and M. J. O'Malley, "A nonlinear mathematical model of electrically stimulated skeletal muscle," IEEE Trans. Rehab. Eng., vol. 5, pp. 179-194, 1997.

[4] S. J. Dorgan and R. Riener, "A physiologically based neuromusculoskeletal model for the design of FNS control strategies," in Proc. 20th Int. Conf. IEEE-EMBS, 1998, pp. 2598-2601.

[5] S. J. Dorgan and M. J. O'Malley, "A mathematical model for skeletal muscle activated by $N$-let pulse trains," IEEE Trans. Rehab. Eng., vol. 6, pp. 286-299, 1998.

[6] L. Gougerot and J. Bulliard, "Non-linéarité des caractéristiques électriques de la peau en courant sinusoïdal de basse fréquence pour des inténsités suffisamment fortes," C. R. Soc. Biol., vol. 144, pp. 768-770, 1950.

[7] W. M. Grill and J. T. Mortimer, "Inversion of the current-distance relationship by transient depolarization," IEEE Trans. Biomed. Eng., vol. 44, pp. 1-9, 1997.

[8] B. Hille, Ionic Channels in Excitable Membranes. Sunderland, MA Sinauer Associates, 1984.

[9] A. L. Hodgkin and A. F. Huxley, "A qualitative description of membrane current and its application to conduction and excitation in nerve," $J$. Physiol., vol. 117, pp. 500-511, 1952.

[10] S. Hozawa, "Studien über die Polarization der Haut. Die 'Anfangszacke' des elektrischen Stromes durch den Menschenkörper," Pflüg. Arch., vol. 219, pp. 110-140, 1928.

[11] R. J. Jaeger, "Lower extremity applications of functional neuromuscular stimulation," Assist. Technol., vol. 4, pp. 19-30, 1992.

[12] K. L. Kilgore, P. H. Peckham, G. B. Thrope, M. W. Keith, and K. A. Gallaher-Stone, "Synthesis of hand grasp using functional neuromuscular stimulation," IEEE Trans. Biomed. Eng., vol. 36, pp. 761-768, 1989. 
[13] R. Kobetic and E. B. Marsolais, "Synthesis of paraplegic gait with multichannel functional neuromuscular stimulation," IEEE Trans. Rehab. Eng., vol. 2, pp. 66-79, 1994.

[14] A. Kralj and T. Bajd, Functional Electrical Stimulation: Standing and Walking after Spinal Cord Injury. Boca Raton, FL: CRC, 1989.

[15] A. Lackermeier, A. Pirke, E. T. McAdams, and J. Jossinet, "Nonlinearity of the skin's AC impedance," in Proc. 18th Int. Conf. IEEE-EMBS, 1997.

[16] D. T. Lykken, "Square wave analysis of skin impedance," Psychophysiol., vol. 7, pp. 262-275, 1970.

[17] J. J. More, "The Levenberg-Marquardt algorithm: Implementation and theory," in Numerical Analysis, Lecture Notes in Mathematics 630, G. A. Watson, Ed. 1977, pp. 105-116.

[18] E. T. McAdams and J. Jossinet, "DC nonlinearity of the solid electrodeelectrolyte interface," Innov. Tech. Biol. Med., vol. 12, pp. 330-342, 1991.

[19] E. T. McAdams and J. Jossinet, "Physical interpretation of Schwan's limit voltage of linearity," Med. Biol. Eng. Comput., vol. 32, pp. 126-130, 1994.

[20] D. McCoy, "A computer controllable stimulator and its use in FES assisted cycle ergometry," M.Eng.Sc. thesis, Nat. Univ. Ireland, 1994.

[21] C. D. Murray, Private Communication, Dec. 1995.

[22] C. D. Murray and R. B. Reilly, "Transcutaneous modeling from voltage and current pulse investigations," Ann. Biomed. Eng., vol. 23, p. S118, 1995.

[23] M. Noshiro, T. Morimoto, H. Nagao, and H. Matsuda, "Electrical impedance in the lower limbs of patients with duchene muscular dystrophy: A preliminary study," Med. Biol. Eng. Comput., vol. 31, pp. 97-102, 1993.

[24] K. S. Osterman, K. D. Paulson, P. J. Hoopes, and A. Hartov, "In vivo electrical impedance spectroscopy of irradiated muscle tissue," in Proc. 19th Int. Conf. IEEE-EMBS, 1997, pp. 2512-2515.

[25] C. J. Poletto and C. van Doren, "A high voltage stimulator for small electrode electrocutaneous stimulation," in Proc. 19th Int. Conf. IEEEEMBS, 1997, pp. 2415-2418.

[26] F. Rattay, "Modeling the excitation of fibers under surface electrodes," IEEE Trans. Biomed. Eng., vol. 35, pp. 199-202, 1994.

[27] H. P. Schwan and C. F. Kay, "Specific resistance of body tissues," Circ. Res., vol. 4, pp. 664-670, 1965 .

[28] R. B. Stein, P. H. Peckham, and D. B. Popovic, Neural Prostheses: Replacing Motor Function after Disease or Disability. New York: Oxford University Press, 1992.

[29] W. G. S. Stephens, "The current-voltage relationship in human skin," Med. Biol. Eng., vol. 1, pp. 389-399, 1963.

[30] A. Varghese, "Membrane models," in The Biomedical Engineering Handbook, J. D. Bronzino, Ed. Boca Raton, FL: CRC, 1995, pp 139-161.

[31] T. Yamamoto and Y. Yamamoto, "Non-linear electrical properties of the skin in the low frequency range," Med. Biol. Eng. Comput., vol. 19, pp. 302-310, 1981

[32] T. Yamamoto and Y. Yamamoto, "Electrical properties of the epidermal stratum corneum,” Med. Biol. Eng., vol. 14, pp. 151-158, 1976.

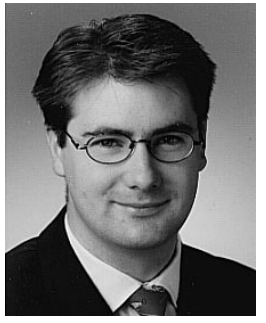

Stephen J. Dorgan (S'94-M'97) was born in Dublin, Ireland, in 1971 and entered University College, Dublin, Ireland (UCD), in 1989. He received the B.E. degree in electronic engineering with first-class honors from the National University of Ireland, Dublin, in 1993. In 1997, he received the $\mathrm{Ph} . \mathrm{D}$. degree in electronic engineering from the same university.

Between 1991 and 1993 he spent time in industry, both in Europe and the United States, working on artificial intelligence and robotic control projects. From 1997 to 1999, he was a Marie Curie Fellow at the Technical University of Munich, Germany, in the Institute of Automatic Control Engineering. He is currently an Associate with McKinsey \& Company, London, U.K. His research interests include nonlinear dynamics, control theory and modeling physiological systems.

Dr. Dorgan is a former Irish Young Scientist first-prize winner, a Founding Member of the International FES Society, a Member of the Royal Academy of Medicine in Ireland, a Member of IEI, and a Member of IEE. While a student at UCD, he was a UCD Presidential Research Scholar, a Lord Edward Fitzgerald Scholar, an Irish-American Partnership Scholar, and a UCD Open Postgraduate Scholar. In 1997, he won the IEE Younger Members Written Paper Premium.



Richard B. Reilly (M'91) received the B.E., M.Eng.Sc., and Ph.D. degrees, all in electronic engineering, from the National University of Ireland, Dublin, in 1987, 1989, and 1992, respectively.

In 1988, he joined Space Technology Ireland, and the Department de Recherche Spatiale, part of the CNRS group, in Paris, France, where he developed a DSP-based Spectrum Analyzer as part of an on-board experiment for the NASA Satellite, WIND. In 1990, he joined the National Rehabilitation Hospital (formerly the National Medical Rehabilitation Centre) as Research Engineer. In 1992, he became a Postdoctoral Research Fellow at University College Dublin, Ireland, where his research interests included nonlinear system identification and modeling, together with alternative and augmentative human-computer interaction, particularly focused on speech enhancement and gesture recognition. He is currently on the academic staff as an Lecturer in the Department of Electronic and Electrical Engineering at University College, Dublin, where is research interests include neurological signal processing, neurocognitive modeling, and human-computer interaction.

Dr. Reilly is an Assistant Editor for the IEEE Transactions on REHABILITATION ENGINEERING. 